\title{
NON-RIGID IMAGE REGISTRATION BY USING GRAPH-CUTS WITH MUTUAL INFORMATION
}

\author{
Ronald W. K. So and Albert C. S. Chung \\ Lo Kwee-Seong Medical Image Analysis Laboratory, \\ Department of Computer Science and Engineering, \\ The Hong Kong University of Science and Technology, Hong Kong \\ \{csswk,achung\}@ cse.ust.hk
}

\begin{abstract}
Non-rigid image registration plays an important role in medical image analysis. Recently, Tang and Chung proposed to model the non-rigid medical image registration problem as an energy minimization framework. The optimization was done by using the graph-cuts algorithm via alpha-expansions. However, the dissimilarity measure used in the energy function of this graph-cuts based method was restricted to the sum of absolute differences (SAD) and the sum of squared differences (SSD). In this paper, to utilize an advanced dissimilarity measure, such as mutual information (MI), we adopt an approximation of MI to the graph-cuts based method. Exploiting the mutual information is valuable as it can capture complex statistical relationships between the intensities of the image pair without a priori knowledge of those relationships. We have compared the proposed method against the original graph-cuts based methods, and two state-of-the-art approaches. The experimental results demonstrate that the proposed method can achieve lower registration errors.
\end{abstract}

Index Terms - Non-rigid image registration, graph cuts, mutual information.

\section{INTRODUCTION}

Non-rigid image registration is widely exploited in medical image analysis. The utilization includes deformation field recovery during surgery, surgical procedure planning and evaluation [1]. All these applications require an accurate registration to improve the precision of diagnosis and therefore nonrigid image registration has been an active research area. The image registration problem can be generally regarded as finding an optimal transformation $T^{*}$ such that the reference image $I_{r}$ and the floating image $I_{f}$ are spatially matched. Technically, it is expressed as $T^{*}=\arg \min _{T} C\left(I_{r}, I_{f}^{T}\right)$, where $C$ is a dissimilarity measure that determines the degree of similarity between $I_{r}$ and $I_{f}^{T}$, and $I_{f}^{T}$ is the transformed floating image. For non-rigid registration, pixels in the floating image can be transformed freely. Therefore, the transformation $T$ is usually represented by a deformation vector field $\mathbf{D}$.
A smoothness penalty $S$ is often introduced to ensure that a smooth resultant transformation is obtained. Finding the optimal transformation can be evaluated as

$$
T^{*}=\arg \min _{T}\left(C\left(I_{r}, I_{f}^{T}\right)+\lambda S(T)\right),
$$

where $\lambda$ is a constant governing the strength of the penalty. Determining $T^{*}$ is challenging due to the requirements of smoothness and the high degrees of freedom. Our previous works [2, 3] have suggested to model the non-rigid medical image registration problem as an energy minimization framework. Graph-cuts algorithm via $\alpha$-expansions [4] was employed as the optimization process in the framework. It was also proven that the graph-cuts based method can give a smooth resultant transformation while the high degrees of freedom can be preserved. The objective of this paper is to improve the accuracy of the registration results by adopting mutual information (MI) into the graph-cut based non-rigid registration method.

\section{GRAPH-CUTS BASED NON-RIGID REGISTRATION}

The graph-cuts based non-rigid registration method (is denoted as GC for the simplicity of description) models the nonrigid registration problem as an energy minimization framework based on the concept of Markov Random Fields (MRF). The framework was defined as

$$
\mathbf{D}^{*}=\arg \min _{\mathbf{D}}\left(E_{d}\left(I_{r}(X), I_{f}^{\mathbf{D}}(X)\right)+\lambda E_{s}(\mathbf{D})\right),
$$

where $\mathbf{D}, E_{d}$ and $E_{s}$ are deformation vector field, data term and smoothness term representing the transformation, the dissimilarity measure and the penalty term respectively, $X$ is the spatial domain of the both images, and $I_{f}^{\mathrm{D}}(X)=I_{f}(X+\mathbf{D})$ is the transformed floating image. The data term is restricted to a sum over pixels which is estimated locally, i.e. $E_{d}=\sum_{x \in X} F\left(x ; I_{r}(X), I_{f}^{\mathbf{D}}(X)\right)$ for some functions $F$ which only depends on a pixel $x$. Therefore, some dissimilarity measures which are calculated globally, like MI, cannot 
be adopted as the data term directly. In [2], SAD was used as the data term and the smoothness term was the derivative of the deformation vector field. Finally, the formula becomes

$$
\mathbf{D}^{*}=\arg \min _{\mathbf{D}}\left(\sum_{x \in X} \operatorname{SAD}\left(I_{r}, I_{f}^{\mathbf{D}}\right)+\lambda \mathbf{D}^{\prime}\right),
$$

where $\operatorname{SAD}\left(I_{r}, I_{f}^{\mathbf{D}}\right)=\sum_{x \in X}\left\|I_{r}(x)-I_{f}^{\mathbf{D}}(x)\right\|$ and $\mathbf{D}^{\prime}=$ $\sum_{(x, y) \in N}\|\mathbf{D}(x)-\mathbf{D}(y)\|$ for $(x, y) \in N$ if $y \in X$ is the adjacent pixel of $x$.

\section{APPROXIMATION OF MUTUAL INFORMATION}

Mutual information (MI) is often applied as the similarity measure in the registration problem [5], in which, MI is defined as

$$
M I\left(I_{r}, I_{f}^{\mathbf{D}}\right)=H\left(I_{r}\right)+H\left(I_{f}^{\mathbf{D}}\right)-H\left(I_{r}, I_{f}^{\mathbf{D}}\right)
$$

where $H(\cdot)$ is the entropy of the given image and $H(\cdot, \cdot)$ is the joint entropy of the given image pair. However, as MI is calculated globally, it cannot be applied to GC directly. In order to achieve this, we use an approximation of MI which was proposed by Kim et al.[6]. The authors express the joint entropy by the Taylor expansion such that $H\left(I_{r}, I_{f}^{\mathbf{D}}\right)=\sum_{x \in X} h\left(I_{r}(x), I_{f}^{\mathbf{D}}(x)\right)$ where

$h\left(I_{r}(x), I_{f}^{\mathbf{D}}(x)\right)=\frac{-1}{|X|}\left(\log \left(P_{I_{r}, I_{f}^{\mathbf{D}}} \otimes g\right) \otimes g\right)\left(I_{r}(x), I_{f}^{\mathbf{D}}(x)\right)$

for $P_{I_{r}, I_{f}^{\mathrm{D}}}$ denoting joint probability distribution and $\otimes g$ indicating 2D Gaussian convolution. Since Kim et al used the approximation in visual correspondence problem, they assume that the $H\left(I_{r}\right)$ and $H\left(I_{f}^{\mathbf{D}}\right)$ are constants during the optimization process. Thus, the approximation of MI is expressed by the joint entropy alone, i.e. $M I\left(I_{r}, I_{f}^{\mathbf{D}}\right)=-H\left(I_{r}, I_{f}^{\mathbf{D}}\right)$. Nevertheless, the assumption does not hold in non-rigid registration problem as $H\left(I_{f}^{\mathbf{D}}\right)$ may change while finding the optimal transformation. As such, we compute the entropies $H\left(I_{r}\right)$ and $H\left(I_{f}^{\mathbf{D}}\right)$ analogous to the joint entropy [7]: $H(I)=\sum_{x \in X} h_{I}(I(x))$, for $h_{I}(I(x))=\frac{-1}{|X|}\left(\log \left(P_{I} \otimes g\right) \otimes\right.$ $g)(I(x))$, where $I$ is an image and $P_{I}$ is the marginal distribution of $I$. Then, the approximation of MI is re-formulated as

$$
\begin{aligned}
& M I\left(I_{r}, I_{f}^{\mathbf{D}}\right)=\sum_{x \in X} \operatorname{mi}\left(I_{r}(x), I_{f}^{\mathbf{D}}(x)\right) \\
& =\sum_{x \in X}\left[h_{I_{r}}\left(I_{r}(x)\right)+h_{I_{f}^{\mathbf{D}}}\left(I_{f}^{\mathbf{D}}(x)\right)-h\left(I_{r}(x), I_{f}^{\mathbf{D}}(x)\right)\right] .
\end{aligned}
$$

\section{GRAPH-CUTS BASED METHOD WITH MI}

It is observed that Eq. 5 is now in the form of sum over pixels. Therefore, we can adopt Eq. 5 into the framework of

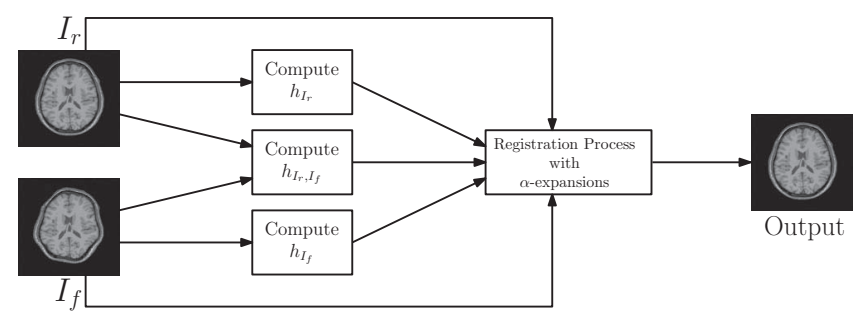

Fig. 1. Flow chat of the proposed method.

GC. The corresponding definition of the energy minimization framework is,

$$
\mathbf{D}^{*}=\arg \min _{\mathbf{D}}\left(\sum_{x \in X}-m i\left(I_{r}, I_{f}^{\mathbf{D}}\right)+\lambda \mathbf{D}^{\prime}\right),
$$

As MI can capture complex relationships between the intensities of the image pair without a priori knowledge of the relationships, it can increase the accuracies of the registration results. Fig. 1 is the flow chat of the framework. Since the entropies $h_{I}(\cdot)$ and $h(\cdot, \cdot)$ do not depend on the transformation, we only need to compute them once and this can finish within a few seconds for a 2D image pair. The pre-computed entropies are then passed to the registration process with $I_{r}$ and $I_{f}$. The process finds the optimal deformation vector field $\mathbf{D}^{*}$ based on minimizing the approximation of MI by graph-cuts algorithm via $\alpha$-expansions [4].

\section{EXPERIMENTAL RESULTS}

In this section, the experimental results of the proposed method will be shown and compared against the original graph-cuts based method and two state-of-the-art methods, which are Free Form Deformation based method (FFD) [9] and DEMONS [8]. We followed the experimental settings as described in [2]. The image pairs used in all experiments were intensity normalized within 0 and 255 , and assumed affinely pre-registered. We used the implementations of FFD and DEMONS available in ITK [10] where $15 \times 15$ control point grid was used in FFD. Implementation of grph-cuts algorithm was done by modifying the the source codes provided by Kolmogorov \& Zabih [11]. For the proposed method (denoted as GC-MI hereafter) and the original graph-cuts based method (denoted as GC-SAD hereafter), the displacement window $W$ was set to $\{0, \pm 1, \pm 2, \ldots, \pm 15\}^{2}$. Thus, the deformation labels (vectors) assigned to pixels were chosen from $31 \times 31$ window. Reference image used in our experiments was the $91^{\text {st }}$ slice of the image \#04 of the Simulated Brain Database [12]. The reference image and its segmented images are shown in Fig. 2. Five floating images were obtained by applying different artificial deformations (Cases A to E) to the reference image which are shown in the left column of Fig. 3. All experiments were performed on an Intel $2.13 \mathrm{GHz}$ dual-core CPU with 3 GB RAM. 


\begin{tabular}{|c|c|c|c|c|c|c|}
\hline \multirow{2}{*}{ Case } & \multirow{2}{*}{ Tissue Class } & \multicolumn{5}{|c|}{ Distributions of Absolute Intensity Errors (Mean \pm SD) } \\
\hline & & Before Registration & FFD & DEMONS & GC-SAD & GC-MI \\
\hline \multirow{4}{*}{ Case A } & $\overline{\mathrm{WM}}$ & $13.5707 \pm 16.2608$ & $4.4435 \pm 4.7523$ & $7.4415 \pm 11.2978$ & $0.3145 \pm 1.5510$ & $0.4484 \pm 1.7329$ \\
\hline & GM & $17.6909 \pm 14.6586$ & $5.3806 \pm 6.0575$ & $9.0305 \pm 11.1348$ & $0.6524 \pm 2.6038$ & $0.5552 \pm 2.0774$ \\
\hline & CSF & $31.6048 \pm 25.1295$ & $7.2099 \pm 7.7194$ & $9.1045 \pm 14.5402$ & $1.3381 \pm 4.9341$ & $0.6937 \pm 2.7217$ \\
\hline & Whole Image & $9.7329 \pm 18.3860$ & $3.3572 \pm 5.6648$ & $3.8116 \pm 8.2376$ & $0.4755 \pm 2.8250$ & $0.4157 \pm 1.7362$ \\
\hline \multirow{4}{*}{ Case B } & $\mathrm{WM}$ & $6.5731 \pm 9.1651$ & $6.2614 \pm 7.6065$ & $2.3301 \pm 3.2015$ & $3.5918 \pm 5.6203$ & $0.7888 \pm 1.9757$ \\
\hline & GM & $10.0813 \pm 12.0606$ & $9.1318 \pm 9.9935$ & $2.7989 \pm 4.1611$ & $4.9365 \pm 6.9476$ & $1.0343 \pm 2.3813$ \\
\hline & CSF & $16.2051 \pm 17.5066$ & $14.6778 \pm 14.7441$ & $3.8172 \pm 5.7646$ & $5.1764 \pm 8.6033$ & $1.0753 \pm 2.8241$ \\
\hline & Whole Image & $6.6147 \pm 15.0480$ & $6.5557 \pm 12.4205$ & $2.3585 \pm 5.4032$ & $2.1051 \pm 5.3806$ & $0.5183 \pm 2.0371$ \\
\hline \multirow{4}{*}{ Case C } & WM & $12.4284 \pm 16.9195$ & $5.2887 \pm 6.9239$ & $5.6849 \pm 10.4597$ & $1.3728 \pm 3.3909$ & $0.8495 \pm 2.3127$ \\
\hline & GM & $15.2166 \pm 17.9072$ & $6.1799 \pm 7.7442$ & $5.7376 \pm 10.3964$ & $1.2019 \pm 3.7910$ & $0.6266 \pm 2.0426$ \\
\hline & CSF & $26.0666 \pm 24.7597$ & $9.4588 \pm 13.3008$ & $10.0482 \pm 17.6691$ & $1.7539 \pm 5.1301$ & $0.7205 \pm 2.3490$ \\
\hline & Whole Image & $9.5187 \pm 18.9494$ & $3.8730 \pm 6.7673$ & $3.5925 \pm 9.1513$ & $0.6852 \pm 2.9006$ & $0.4135 \pm 1.7523$ \\
\hline \multirow{4}{*}{ Case D } & WM & $15.2038 \pm 18.7338$ & $6.5360 \pm 8.3473$ & $6.8279 \pm 11.2505$ & $1.2181 \pm 3.1834$ & $1.6124 \pm 3.1227$ \\
\hline & GM & $20.5996 \pm 21.0016$ & $8.2451 \pm 9.4451$ & $6.7825 \pm 11.1975$ & $1.3924 \pm 4.0410$ & $1.1491 \pm 3.1137$ \\
\hline & CSF & $32.9635 \pm 25.8792$ & $12.4072 \pm 12.8791$ & $10.6003 \pm 16.1336$ & $2.3172 \pm 5.9184$ & $1.3010 \pm 3.7120$ \\
\hline & Whole Image & $13.2306 \pm 23.7698$ & $5.5561 \pm 9.8258$ & $4.8540 \pm 11.6549$ & $1.2848 \pm 4.4154$ & $0.9427 \pm 3.7402$ \\
\hline \multirow{4}{*}{ Case E } & WM & $14.3048 \pm 20.5277$ & $7.5955 \pm 9.2995$ & $6.2710 \pm 12.6154$ & $3.5391 \pm 5.6802$ & $2.1649 \pm 3.4320$ \\
\hline & GM & $19.4661 \pm 22.1715$ & $9.7916 \pm 10.5114$ & $8.2438 \pm 14.9239$ & $4.4782 \pm 7.1524$ & $2.6636 \pm 4.2757$ \\
\hline & $\mathrm{CSF}$ & $29.7475 \pm 24.1374$ & $15.3230 \pm 15.3757$ & $11.5549 \pm 17.2870$ & $5.8027 \pm 9.4507$ & $3.5900 \pm 5.8147$ \\
\hline & Whole Image & $15.2396 \pm 26.4708$ & $7.2976 \pm 12.1217$ & $7.3590 \pm 17.9116$ & $2.7162 \pm 5.9417$ & $2.5092 \pm 6.5669$ \\
\hline \multirow{4}{*}{ All } & WM & $12.4162 \pm 16.3214$ & $6.0250 \pm 7.3859$ & $5.7111 \pm 9.7650$ & $2.0073 \pm 3.8852$ & $1.1728 \pm 2.5152$ \\
\hline & GM & $16.6109 \pm 17.5599$ & $7.7458 \pm 8.7503$ & $6.5187 \pm 10.3627$ & $2.5323 \pm 4.9072$ & $1.2058 \pm 2.7781$ \\
\hline & CSF & $27.3175 \pm 23.4825$ & $11.8153 \pm 12.8038$ & $9.0250 \pm 14.2789$ & $3.2776 \pm 6.8073$ & $1.4761 \pm 3.4843$ \\
\hline & Whole Image & $10.8673 \pm 20.5248$ & $5.3279 \pm 9.3600$ & $4.3951 \pm 10.4717$ & $1.4533 \pm 4.2927$ & $0.9599 \pm 3.1665$ \\
\hline
\end{tabular}

Table 1. Distributions of the absolute intensity errors within different tissue regions after performing FFD, DEMONS, GC-SAD and GC-MI. Distributions are listed in the format $\mathrm{MEAN} \pm \mathrm{SD}$.

Columns 2-5 of Fig. 3 show the registration results of FFD, DEMONS, GC-SAD and GC-MI respectively. As shown in the figure, DEMONS performed worse in Cases $\mathrm{C}-\mathrm{E}$ as it allows high-degree of freedom and uses local intensity gradient to optimize the pixel movements. Therefore, for some cases, like Cases C-E, in which some brain tissues of the reference image are mapped to the background of the floating image or vice versa, pixels can be driven towards the wrong directions and DEMONS may be trapped in local minima. On the other hand, FFD performed worse in Cases $\mathrm{B}$ and $\mathrm{E}$ since it uses hard constrains on the pixel movements. In FFD, only control points can be moved freely. The displacements of all other pixels are interpolated from its neighborhood control points by using B-splines. Therefore it is hard for FFD to model complicated deformations like Cases B and D which include high frequency deformations. DEMONS worked well in Case B because of the high degrees of freedom. From Fig. 3, we notice that GC-MI and GC-SAD gave successful registration results in all the cases. The main reason is that they optimize the registration process by using the alpha-expansions which assign a displacement label (vector) to each pixel in global manner with high-degrees of freedom.

Table 1 is the quantitative validation. It shows distributions of the absolute intensity errors within white matter (WM), gray matter (GM), cerebrospinal fluid (CSF) and the whole image after performing FFD, DEMONS, GC-SAD and GC-MI. The distributions are listed in the format of MEAN $\pm \mathrm{SD}$. By comparing the results in the table, it is found that GC-MI and GC-SAD have better performances than FFD and DEMONS whereas GC-MI outperforms GC-SAD. The averaged absolute intensity errors in the last row demonstrate that GC-MI improved the accuracies about 50 100\% among different tissues as compared with GC-SAD.

\section{CONCLUSION}

In this paper, we present the graph-cuts based non-rigid registration method by adopting an approximation of mutual information as the dissimilarity measure. Experimental results have demonstrated that the proposed method is robust against different challenging registration cases. The quantitative results have also shown that the proposed method can improve the averaged absolute intensity errors by $50 \sim 100 \%$. With the proposed techniques, we believe that other dissimilarity measures, such as normalized mutual information, can be employed in the graph-cuts based non-rigid registration method. In the future, more qualitative validations will be conducted, especially in volumetric images, for the proposed method.

\section{REFERENCES}

[1] D. Rueckert, "Non-rigid registration: Techniques and applications," in Medical Image Registration, CRC Press, 2001.

2] Tommy W. H. Tang and Albert C. S. Chung, "Non-rigid image registration using graph-cuts," MICCAI LNCS, vol. 4791, pp. 916-924, 2007.

[3] Ronald W. K. So and Albert C. S. Chung, "Multi-level non-rigid image registration using graph-cuts," in ICASSP, pp. 397-400, 2009.

[4] Y. Boykov, O. Veksler et al, "Fast approximate energy minimization via graph cuts," PAMI, vol. 23 (11), pp. 1222-1239, 2004.

[5] J. P. W. Pluim, J. B. A. Maintz et al, "Mutual-information-based registration of medical images: a survey," T-MI, vol. 22 (8), pp. 986-1004, 2003.

[6] J. Kim, V. Kolmogorov et al, "Visual correspondence using energy minimization and mutual information," in ICCV, 2003.

[7] H. Hirschmüller, "Accurate and efficient stereo processing by semi-global matching and mutual information," in CVPR, 2005.

[8] J. Thirion, "Image matching as a diffusion process: an analogy with maxwell's demons," MedIA, vol. 2 (3), pp. 243-260, 1998.

[9] D. Rueckert, L. I. Sonoda et al, "Non-rigid registration using free-form deformations: Application to breast $\mathrm{mr}$ images," $T-M I$, vol. 18 (8), pp. 712-721, 1999.

[10] L. Ibanez, W. Schroeder et al., The ITK Software Guide, 2003.

[11] V. Kolmogorov and R. Zabih, "What energy functions can be minimized via graph cuts?," PAMI, vol. 26 (2), pp. 147-159, 2005.

[12] B. Aubert-Broche, A. Evans et al, "A new improved version of the realistic digital brain phantom," NeuroImage, vol. 32 (1), pp. 138-145, 2006. 


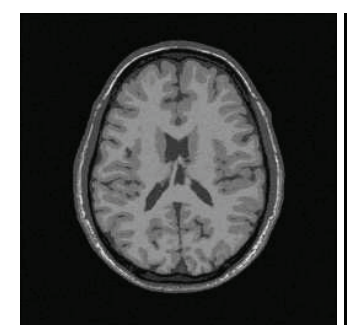

Reference Image

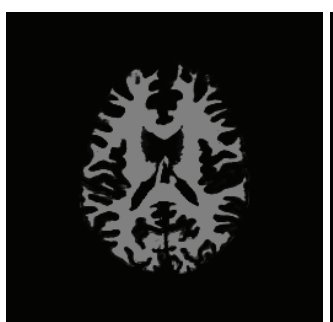

WM

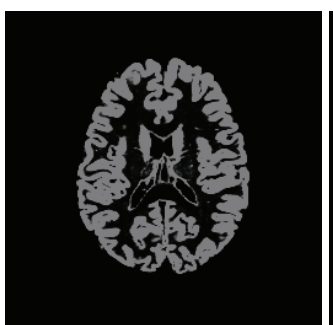

GM

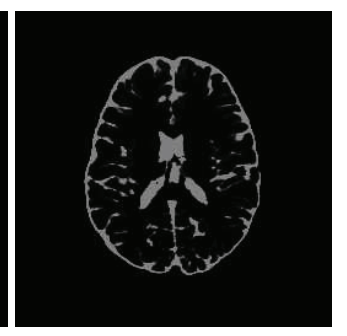

CSF

Fig. 2. Reference and its segmented images obtained from Simulated Brain Database used in our experiments.

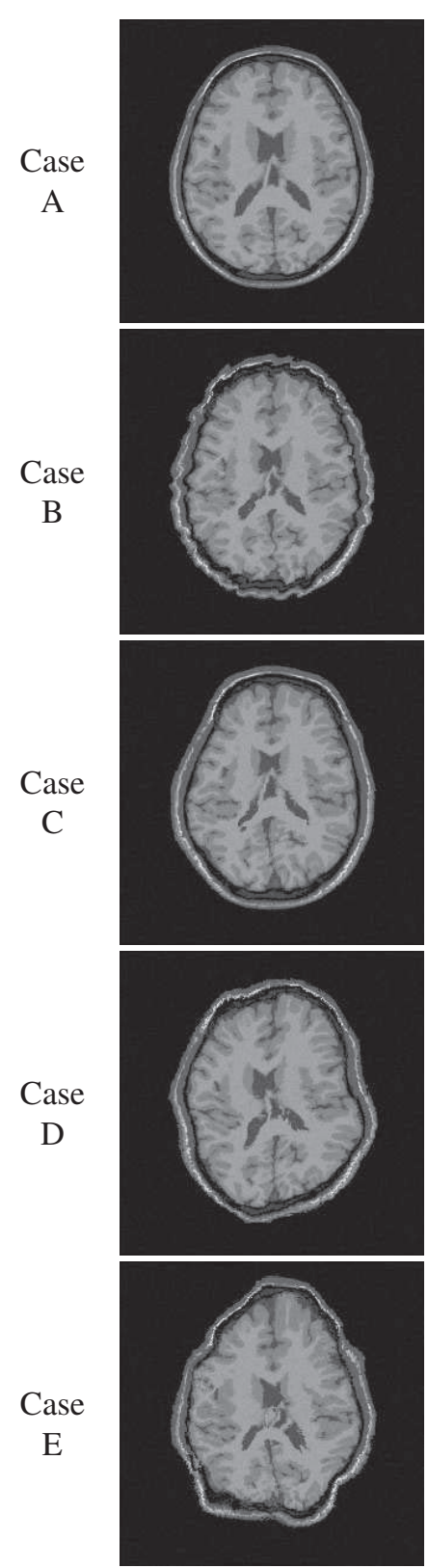

Floating
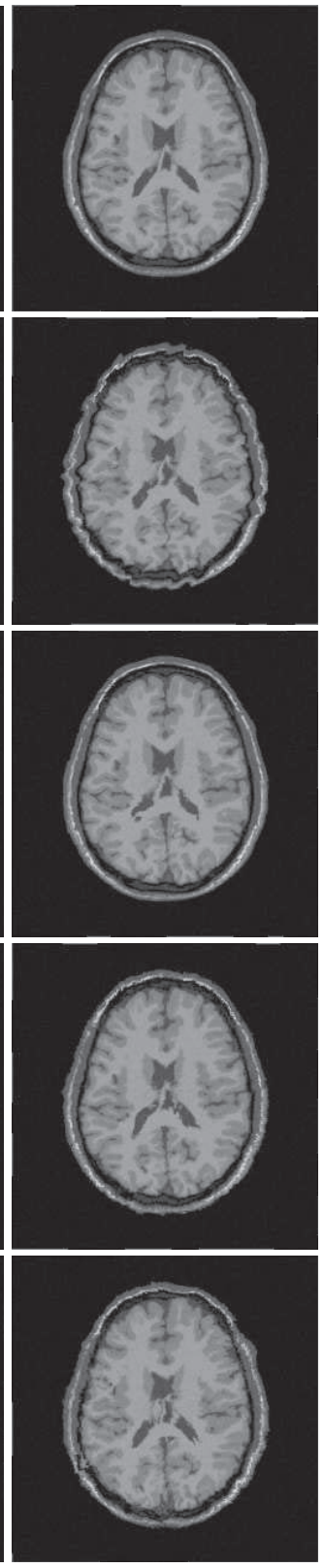

FFD
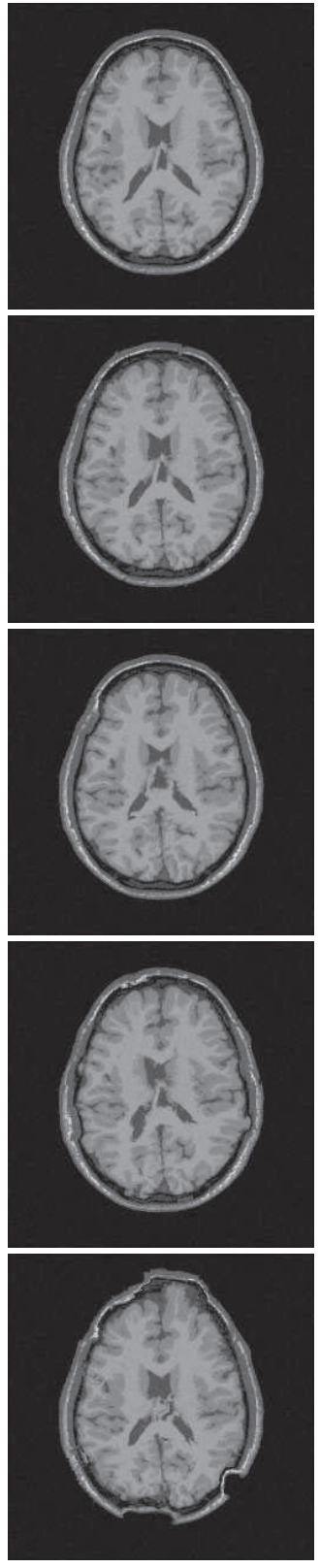

DEMONS
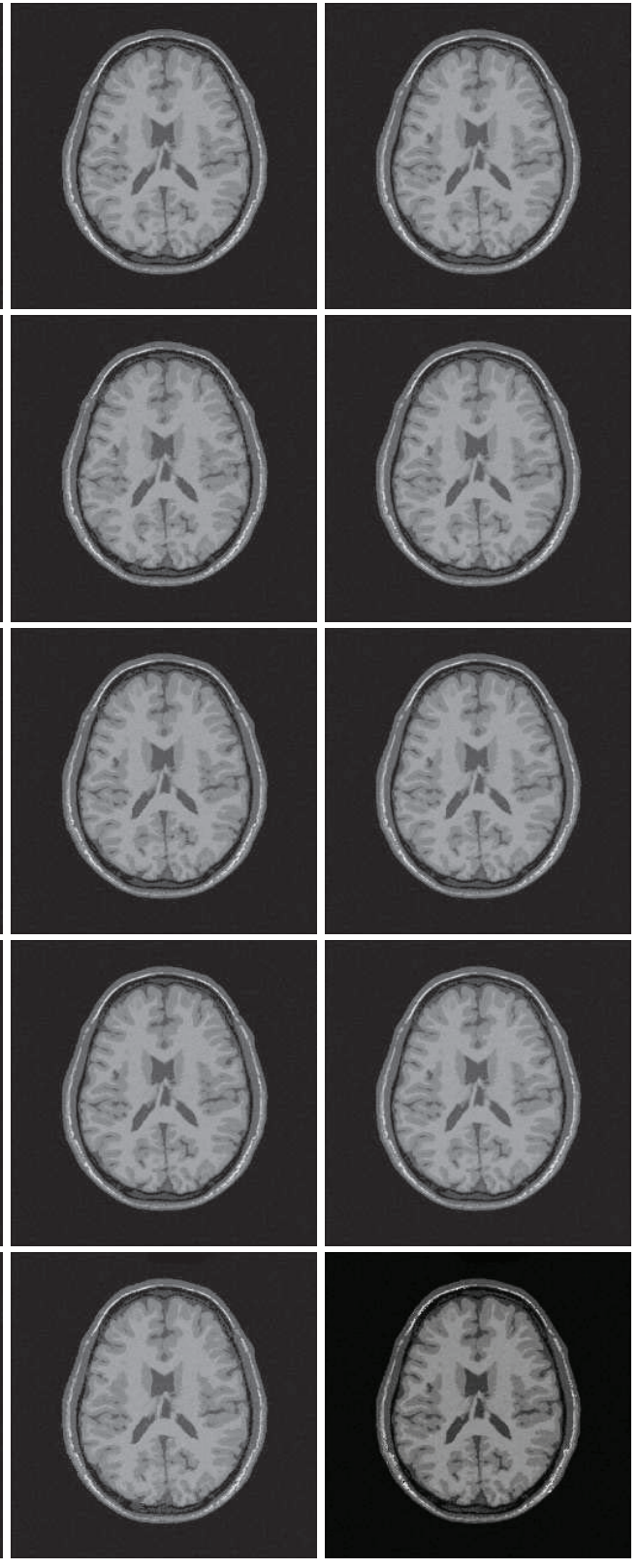

GC-SAD

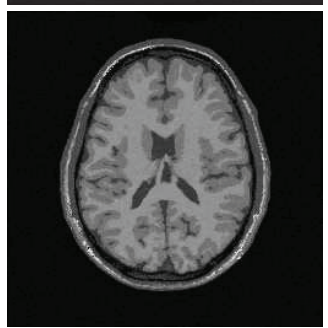

GC-MI

Fig. 3. Results of 5 different artificial deformation cases. 